\title{
Early coronary stent thrombosis after bare metal stent implantation
}

\section{Dubey L, ${ }^{1}$ Guruprasad S, ${ }^{2}$ Subramanyan $\mathbf{G}^{3}$}

${ }^{1}$ Resident DM Cardiology, ${ }^{2}$ Associate Professor, ${ }^{3}$ Professor and HOD, Department of Cardiology, College of Medical Sciences and Teaching Hospital, Bharatpur, Chitwan, Nepal

\section{ABSTRACT}

Percutaneous coronary intervention with stenting has become the most widely performed procedure for the treatment of obstructive coronary artery disease. But, a major drawback of coronary stenting is the occurrence of stent thrombosis. Though rare, stent thrombosis can lead to ST segment elevation myocardial infarction or even sudden cardiac death. This is a case of early coronary stent thrombosis in a 58-year-old male patient, who presented with acute chest pain, Mobitz type II 2:1atrioventricular block and cardiogenic shock 15 days after percutaneous coronary intervention with bare metal stenting to the right coronary artery.

Key Words: Acute myocardial infarction; coronary stenting; bare metal stent; early stent thrombosis

\section{INTRODUCTION}

Percutaneous coronary intervention (PCI) with stenting with bare metal stent (BMS) or drug eluting stent (DES) has become the most widely performed procedure for the treatment of obstructive coronary artery disease. Stent implantation leads to activation of the coagulation cascade which may lead to stent thrombosis (ST). ${ }^{1}$ Though rare, the consequences of ST can be fatal, as most ST cases are associated with myocardial infarction or sud-den death. ${ }^{2}$ ST can be divided into early ST (0 to 30 days), late ST (31days to 1 year), and very late ST ( $>1$ year). ${ }^{3}$ In case of BMS, complete stent endothelization usually occurs after one month. After this period it is very uncommon to encounter ST in BMS. Early ST may be related to non-adherence to antiplatelet agents, residual target lesion thrombus or dissection,

Correspondence to: Dr. Laxman Dubey

E-mail: dubeylax@yahoo.com stasis, stent underexpansion, or a combination of these. In this paper, a case of early ST that occurred after 15 days following BMS implantation is reported.

\section{CASE REPORT}

A 58-year-old man with a history of hypertension and present smoker presented to our emergency department with a complaint of acute central chest pain for three hour duration which was associated with nausea and 2 episodes of vomiting. He also had one episode of sudden loss of consciousness. On presentation, he was conscious. His blood pressure (BP) was $80 / 50 \mathrm{mmHg}$ and pulse rate of $40 \mathrm{bpm}$. Electrocardiagraphy (ECG) revealed Mobitz type II 2:1 atrioventricular (AV) block with ST segment elevation in leads II, III and aVF with qS pattern in leads V1 and V2 and ST segment depression with T wave inversion in leads V4-V6. A loading dose of 300 $\mathrm{mg}$ aspirin and $600 \mathrm{mg}$ clopidogrel was given. Patient was taken to the 
Dubey L et al. Early coronary stent thrombosis after bare metal stent implantation

catheterization laboratory where coronary angiography revealed subtotal occlusion of the left anterior descending (LAD) artery and total occlusion of the right coronary artery (RCA) at the proximal level (figure 1A). Primary PCI to the RCA (culprit vessel) was planned. PCI was approached via right femoral artery. Temporary pacemaker was inserted via right femoral vein and kept in the right ventricle. RCA was hooked with Judkins Right guiding catheter $3.5 \times 6 \mathrm{~F}$ (Cordis). Lesion crossed with a BMW guidewire (Abbott vascular). After guidewire crossing the lesion, antegrade flow in the RCA was seen. Lesion was predilated with Sprinter balloon (Medtronic) measuring $2 \times 10 \mathrm{~mm}$ at 12 Atm, followed by the deployment of a cobaltchromium BMS (Presillion Plus, Medtronic) measuring $3 \times 33 \mathrm{~mm}$ at $14 \mathrm{Atm}$. The final result was deemed satisfactory with TIMI III flow (figure 1B). Post procedure BP was $100 / 60$ on intravenous inotropic support and normal saline; however, AV block reverted to normal sinus rhythm. Temporary pacemaker was removed.

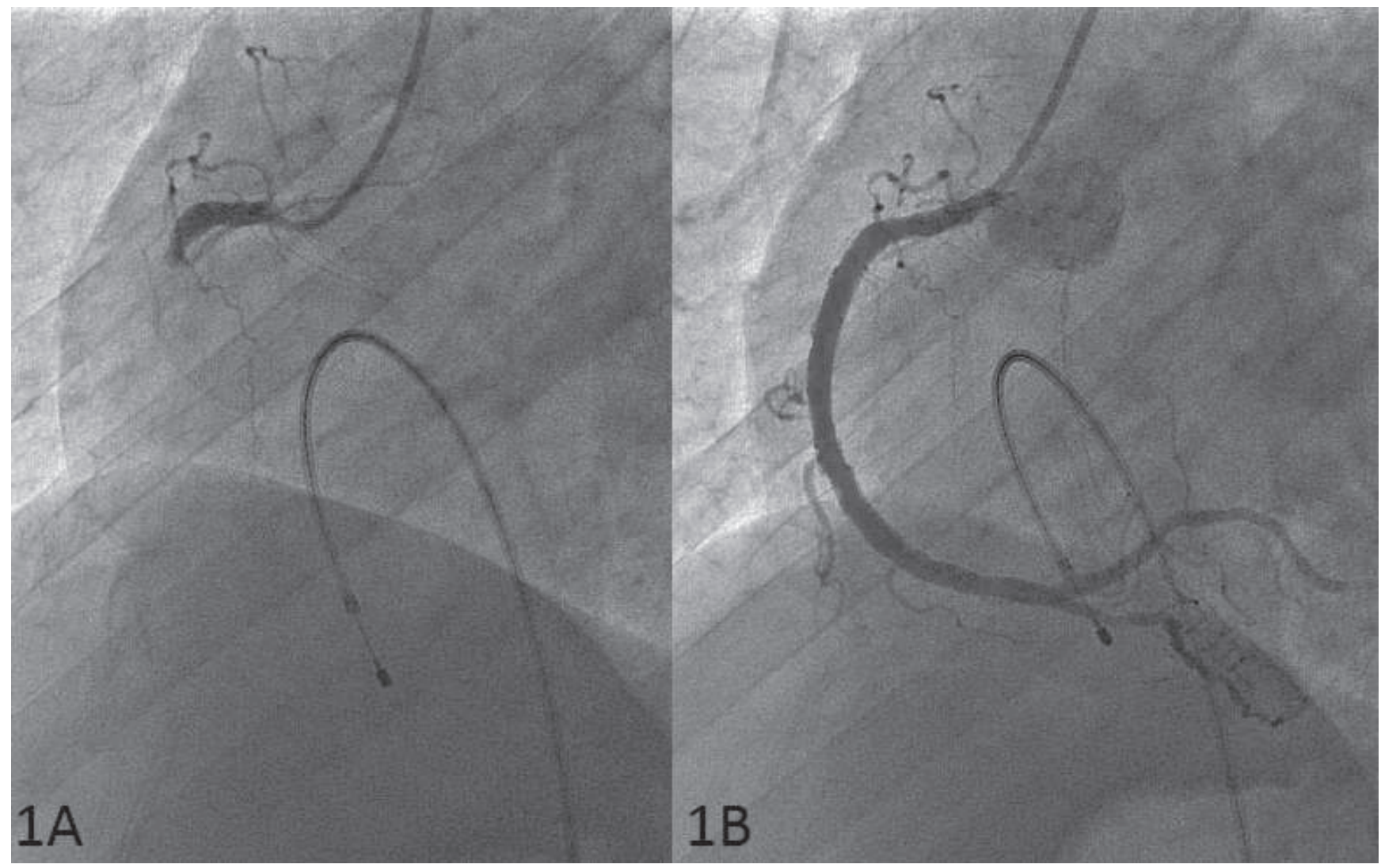

Figure 1A. Selective coronary angiography of the right coronary artery at left anterior oblique projection showed total occlusion at the proximal part

Figure 1B. Final angiographic result after stent implantation (TIMI III flow)

Patient was transferred to the coronary care unit and was under inotropic support of dopamine and dobutamine. During his subsequent stay in the hospital, he received low molecular weight heparin, enteric coated aspirin, clopidogrel, atorvastatin and ramipril. Patient's hospital stay was uneventful and discharged after 3 days.

Four days later, patient visited our out-patient department for follow up and he stated that he is 
Journal of College of Medical Sciences-Nepal, 2013, Vol-9, No-2,

comfortable and free of angina. His BP was 120/ $70 \mathrm{mmHg}$, pulse of $70 \mathrm{bpm}$. ECG showed sinus rhythm at $72 \mathrm{bpm}$, QS with $0.5 \mathrm{~mm}$ ST segment elevation in leads III and $\mathrm{aVF}$ and T wave inversion in leads II, III and aVF. PR interval was $160 \mathrm{msec}$. Previous medications were continued and bisoprolol was added. Eight days later he was brought to our emergency department with acute chest pain for 1 hour associated with three episodes of vomiting and one episode of loss of consciousness. On examination, patient was conscious with no focal neurological deficit. His BP was $70 / 50 \mathrm{mmHg}$, pulse of $40 \mathrm{bpm}$ with ongoing chest pain. ECG showed Mobitz type II 2:1 AV block with ventricular rate of $42 \mathrm{bpm}$. There was QS pattern with $2 \mathrm{~mm}$ ST segment elevation in leads III and aVF and $1 \mathrm{~mm}$ ST segment elevation in lead II with $\mathrm{T}$ wave inversion. He mentioned that he was not taking any medications since last 7 days. Since his ECG showed increased ST segment elevation with new onset AV block, stent thrombosis (ST) was suspected and patient transferred to the catheterization laboratory. Inotropes and intravascular normal saline were given intravenously. A temporary pacemaker was placed in the right ventricle. Selective coronary angiography via right femoral approach showed total occlusion of the RCA (figure 2). A diagnosis of early ST was made. Lesion was crossed with a BMW guidewire but no antegrade flow was seen. Balloon dilation was done using Sprinter semicomplaint balloon measuring $2.0 \times 10$ at 14 ATM, and again with $2.5 \times 12$ balloon at 18 ATM. But no antegrade flow was noted. In the meantime, patient developed ventricular tachycardia which was reverted with DC shock. Then patient become restless and went into asystole. Chest compression was started and patient was intubated. No flow in the RCA was noted and the CPR continued. Patient revived after CPR and transferred to the coronary care unit. Next day, patient's general condition improved, blood pressure was 130/80 $\mathrm{mmHg}$, pulse of $90 \mathrm{bpm}$. Patient was extubated, inotopic agents were tapered and temporary pacemaker was removed. Fortunately, patient's further hospital stay was uneventful and discharged on seventh day of admission. Transthoracic echocardiography revealed akinetic anterior and inferior segments, moderate mitral regurgitation with severe LV systolic dysfunction (LVEF 25\%). No further interventions were done and the patient was kept on triple antiplatelet agents (aspirin, clopidogrel and cilostazol). At three month follow-up, patient doing fine.

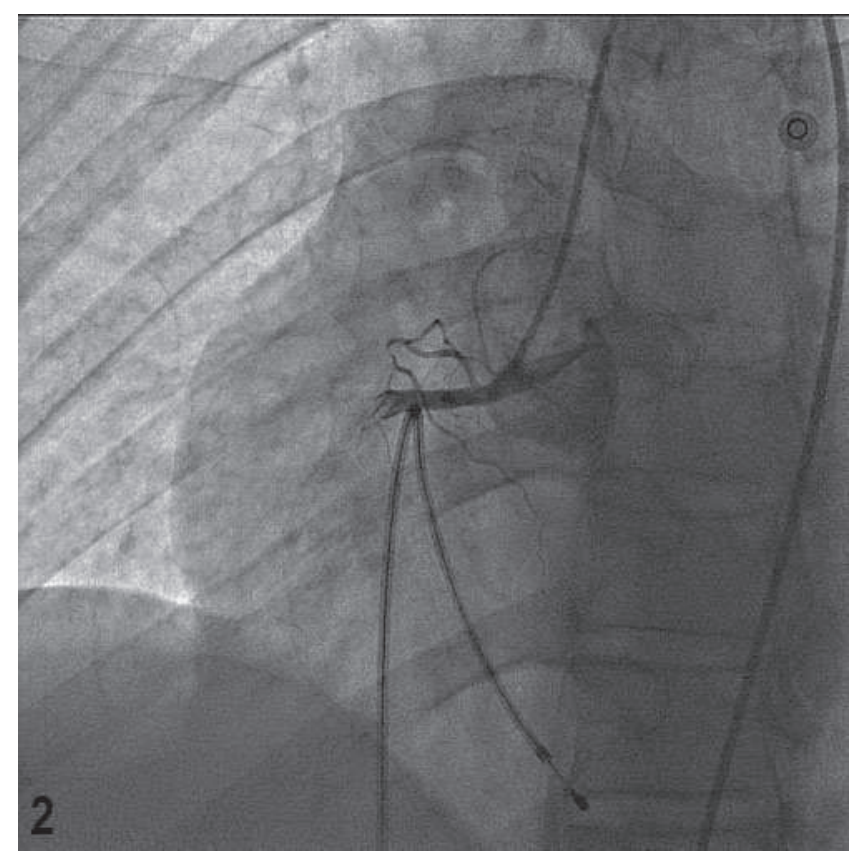

Figure 2. Selective coronary angiography of the right coronary artery showed stent thrombosis with total occlusion

\section{DISCUSSION}

Stents were introduced as a bailout procedure in cases of threatened vessel closure ${ }^{4}$ which soon revolutionized the practice of interventional cardiology as stent implantation dramatically improved upon the success rate for the initial 
Dubey L et al. Early coronary stent thrombosis after bare metal stent implantation

procedure, as well as a lower restenosis rate at midterm follow up. ${ }^{5,6}$ Despite these advantages, a major drawback was the relatively common occurrence of ST. Coronary artery ST is a rare complication, mostly presenting as ST segment elevation myocardial infarction or sudden cardiac death. ${ }^{2}$ During PCI and stent implantation, vascular injury leads to adherence of activated platelets at the site of injury. There is release of adenosine diphosphate, tromboxane A2 and other procoagulative factors. Release of these factors further leads to platelet aggregation and the formation of platelet-rich thrombi. ST occurs in less than $1 \%$ of BMS $^{7}$ and most cases of ST occur within the first month after deployment, irrespective of the stent type. ${ }^{8}$ In TAXUS V trial, the incidence of early ST ( $<30$ days) in the baremetal arm of the study group was $0.5 \%$. ${ }^{9}$ Several factors related to the patient, coronary lesion and percutaneous intervention predispose to ST. These factors include noncompliance with antiplatelet agents, exercise-induced procoagulant state, brachytherapy, small stent size and underdeployment of the stent, impaired response to antiplatelet therapy and residual thrombus or persistent dissection after stent placement.

In our patient, the time to the acute thrombotic event from the index PCI was 15 days. Since he was not on dual antiplatelet therapy, we could assume that non-adherence to the antiplatelet agents could be the most important predictor for ST in our patient. Another potential explanation for this acute event in our patient is the possibility of stent malapposition. It has been reported that the most common cause of early bare-metal ST is stent malapposition. ${ }^{10}$ This can be attributed to dissection at the edges of the stent or stent deployment issues such as incomplete expansion and apposition. However, since intravascular ultrasound was not performed because of its unavailability in our center, this suspicion could not be confirmed. Another possibility for early ST could be impaired response to antiplatelet therapy.

In conclusion, early ST with the use of BMS, although fortunately infrequent, is a catastrophic event. Avoidance of stent undersizing by the operator, regular use of higher balloon pressures during stent implantation and strict adherence to dual antiplatelet treatment are crucial to minimize this complication.

\section{ACKNOWLEDGEMENT}

Authors would like to thank Dr. SK Sharma and catheterization laboratory technician Mr. I Dumre for their assistance during the procedure.

\section{REFERENCES}

1. Lee MS, David EM, Makkar RR, et al. Molecular and cellular basis of restenosis after percutaneous coronary intervention: The intertwining roles of platelets, leukocytes, and the coagulationfibrinolysis system. J Pathol 2004;203:861-70.

2. Iakovou I, Schmidt T, Bonizzoni E, et al. Incidence, predictors, and out-come of thrombosis after successful implantation of drug-eluting stents. JAMA 2005;293:2126-30.

3. Cutlip DE, Windecker S, Mehran R, et al. Clinical end points in coronary stent trials: a case for standardized definitions. Circulation 2007;115:2344-51.

4. Sigwart U, Puel J, Mirkovitch V, et al. Intravascular stents to prevent occlusion and restenosis after transluminal angioplasty. $N$ Engl $J$ Med 1987;316:701-6. 
Journal of College of Medical Sciences-Nepal, 2013, Vol-9, No-2,

5. Fischman DL, Leon MB, Baim DS, et al. A randomized comparison of coronary-stent placement and balloon angioplasty in the treatment of coronary artery disease. Stent Restenosis Study Investigators. N Engl J Med 1994;331:496-501.

6. Serruys PW, de Jaegere P, Kiemeneij F, et al. A comparison of balloon-expandable-stent implantation with balloon angioplasty in patients with coronary artery disease. Benestent Study Group. N Engl J Med 1994;331:489-95.

7. Cutlip DE, Baim DS, Ho KK, et al. Stent thrombosis in the modern era: A pooled analysis of multicenter coronary stent clinical trials. Circulation 2001;103:1967-71.
8. Aoki J, Lansky AJ, Mehran R, et al. Early stent thrombosis in patients with acute coronary syndromes treated with drug-eluting and bare metal stents: The Acute Catheterization and Urgent Intervention Triage Strategy trial. Circulation 2009; 119:687-98.

9. Stone GW, Ellis SG, Cannon L, et al; TAXUS V Investigators. Comparison of a polymer-based paclitaxel-eluting stent with a bare metal stent in patients with complex coronary artery disease: a randomized controlled trial. JAMA 2005; 294:121523

10. Lampropoulos KM, Iliopoulos TA, Budts W. Early bare-metal stent thrombosis presenting with cardiogenic shock: a case report. J Med Case Rep 2011,5:509 